Service social

\title{
Prison et ordre social au Québec, par Jacques Laplante, Ottawa, Les Presses de l’Université d'Ottawa, 1989, 238 pages.
}

\section{Germain Trottier}

Volume 39, numéro 2, 1990

Les problèmes sociaux

URI : https://id.erudit.org/iderudit/706494ar

DOI : https://doi.org/10.7202/706494ar

Aller au sommaire du numéro

Éditeur(s)

École de service social de l'Université Laval

ISSN

1708-1734 (numérique)

Découvrir la revue

Citer ce compte rendu

Trottier, G. (1990). Compte rendu de [Prison et ordre social au Québec, par Jacques Laplante, Ottawa, Les Presses de l'Université d'Ottawa, 1989, 238 pages.] Service social, 39(2), 251-252. https://doi.org/10.7202/706494ar d'utilisation que vous pouvez consulter en ligne.

https://apropos.erudit.org/fr/usagers/politique-dutilisation/ 
même une diminution du pouvoir excessif de ce dernier. On demande aussi la possibilité de bénéficier d'approches non médicales, de centres de santé pour les femmes, de thérapies féministes. Les femmes elles-mêmes devraient changer leurs habitudes de vie, apprendre à se mobiliser et à aller chercher l'information.

Cet ouvrage présente un constat de la situation de santé actuelle des Québécoises, vue sous divers angles par des femmes reconnues dans leur domaine respectif. Fait à noter : bien qu'on assiste de plus en plus à une féminisation de la profession médicale, on ne retrouve ici aucun article écrit par l'une de ces femmes. De même, il aurait sans doute été intéressant de lire les attentes, en matière de santé, des utilisatrices elles-mêmes. II me vient aussi un certain questionnement face au choix ou plutôt au non-choix des thèmes; par exemple, on passe à peu près sous silence les problèmes de santé mentale, les MTS, les maladies liées au vieillissement, la fatigue des femmes, I'usage des drogues et de l'alcool, les agressions sexuelles et incestueuses, etc. Cependant, pour les sujets traités et pour les avenues futures, les intervenants et intervenantes gagneront à la lecture de cette publication : les hommes, pour mieux comprendre, et les femmes, pour se souvenir que les acquis, dans ce domaine comme ailleurs, sont toujours fragiles et surtout rarement gratuits.

Sylvie Berger

Infirmière-chef, Département d'urgence

Centre hospitalier de l'Université Laval

\section{Prison et ordre social au Québec, par Jacques LAPLANTE, Ottawa, Les Presses de I'Université d'Ottawa, 1989, 238 pages.}

Il s'agit d'un ouvrage publié à la suite d'une recherche universitaire qui se proposait d'étudier le système de justice pénale en milieu québécois et plus particulièrement d'examiner l'institution de la prison comme moyen de répression des pauvres, couche sociale défavorisée et surreprésentée dans les milieux carcéraux.

À cette fin, I'auteur a rédigé sa publication en trois parties, qui correspondent à toutes fins pratiques à trois périodes significatives de l'histoire du Québec. La première partie, qui couvre la période 1608-1830, explique de façon détaillée le fonctionnement naturel de la justice autochtone des Amérindiens, puis l'imposition successive des mesures pénales sous les régimes français et anglais.

Dans la seconde partie, correspondant à la période 1830-1930, l'auteur tente de démontrer comment $s^{\prime}$ est progressivement construit le système pénal et à quel point la prison est devenue un moyen organisé pour réprimer la population cible des pauvres au détriment des autres couches sociales.

Enfin, dans la troisième et dernière partie couvrant la période de 1930 à nos jours, I'ouvrage analyse la phase de consolidation du système, le mouvement des réformes et les finalités thérapeutiques introduites dans le discours pour justifier le maintien et le développement de la prison, au nom de l'ordre et de la défense sociale.

Pour guider sa démarche d'analyse tout au long de l'ouvrage, l'auteur fait appel à des paramètres qu'il nomme "les états fondamentaux de comman- 
dement, de culte, de production et échange " pour expliquer l'existence du lien constant entre la prison et la pauvreté.

L'utilisation de la méthode historique est à notre avis un procédé judicieux et original. En même temps que l'auteur fournit une source crédible de références, son œuvre prend l'allure d'un récit qui peut intéresser même les néophytes du domaine de la criminologie. Le vocabulaire choisi est simple, le style est clair, l'écriture est sans ambiguïté, la démarche est logique et convaincante. Le caractère d'impression, serré, est toutefois à déplorer n'étant pas de nature à faciliter la lecture du volume.

Par les aspects historiques soulevés, l'ouvrage suscitera certainement de l'intérêt chez les professionnels et ex-professionnels du milieu carcéral, mais peut-être aussi un certain malaise ou un requestionnement critique parce que le volume révèle des manigances exercées bien souvent à leur insu ou en l'absence de connaissances suffisantes.

La force véritable de ce volume réside dans le fait qu'il met à jour, pour les présentes générations de professionnels des services correctionnels, les enjeux qui ont originé et qui persistent dans le système de justice pénale québécoise. Dans ce sens, l'ouvrage du professeur Laplante constitue un outil remarquable d'enseignement et de formation en matière de pénologie et de politique criminelle au Québec. Tout lecteur est incité à développer un questionnement critique et conscient sur le sens et la portée de la prison comme institution. Si tel était le but de l'auteur, l'objectif est atteint.

La dernière portion de la troisième partie du volume, titrée "la prison au cœur du social », nous interroge par rapport au reste de l'ouvrage. En dépit de restrictions annoncées, on ne sait trop s'il s'agit d'une fin mal bouclée, de l'amorce d'une conclusion qui reste inachevée ou d'une stratégie de l'auteur qui veut inciter le lecteur à se positionner. L'impression qui en reste est floue et donne malheureusement à penser que l'auteur a manqué de souffle pour conclure, ce qui enlève un peu de fini au volume qui se présentait jusqu'ici comme un travail remarquable.

Outre cette dernière remarque de portée mineure, il s'agit d'un excellent ouvrage critique sur le système de justice en contexte québécois, à recommander pour lecture et usage à des fins d'enseignement.

Germain Trottier

École de service social

Université Laval

\section{Projet "Chômeurs Créateurs Associés ", par Marie-Chantal} GUÉDON, Saint-Jean-sur-Richelieu, Centre de formation à I'Autogestion du Haut-Richelieu (C.P. 532, Saint-Jean-surRichelieu, Québec, J3B 6Z4), 1989, 84 pages.

Cette monographie décrit et évalue une intervention menée entre février 1986 et octobre 1988, auprès de jeunes sans emploi, de 18 à 30 ans, de la région de Saint-Jean-sur-Richelieu. L'approche retenue était celle du "chômage créateur ", défini comme une " attitude consistant à dépasser l'attente passive ou la recherche effrénée d'un emploi pour exploiter le temps libéré par l'absence d'emploi et le remplir par des activités ou des moments de réflexion susceptibles de favoriser un meilleur épanouissement et une plus grande prise 